\title{
MECHANISMS FOR DIRECT BREAKUP REACTIONS ${ }^{\dagger}$
}

\author{
G. BAUR \\ Forschungszentrum Jülich, Institut für Kernphysik, D-52425 Jülich, Germany \\ E-mail: g.baur@fz-juelich.de \\ S. TYPEL and H. H. WOLTER \\ Sektion Physik, Universität München, D-85748 Garching, Germany \\ E-mail: stefan.typel@physik.uni-muenchen.de, \\ hermann.wolter@physik.uni-muenchen.de \\ K. HENCKEN and D. TRAUTMANN \\ Institut für Theoretische Physik, Universität Basel, \\ Klingelbergstraße 82, CH-4056 Basel, Switzerland \\ E-mail: k.hencken@unibas.ch, \\ dirk.trautmann@unibas.ch
}

\begin{abstract}
We review some simple mechanisms of breakup in nuclear reactions. We mention the spectator breakup, which is described in the post-form DWBA. The relation to other formulations is also indicated. An especially important mechanism is Coulomb dissociation. It is a distinct advantage that the perturbation due to the electric field of the nucleus is exactly known. Therefore firm conclusions can be drawn from such measurements. Some new applications of Coulomb dissociation for nuclear astrophysics are discussed.
\end{abstract}

\section{Introduction}

In general, the dynamics of nuclear breakup reactions can be quite complicated. We wish to discuss in this minireview style some limiting cases, which show some simple features. One may regard the work of Oppenheimer and Phillips in $1935 \mathrm{H}$ a a a starting point of the present subject. They tried to explain the preponderance of $(\mathrm{d}, \mathrm{p})$-reactions over $(\mathrm{d}, \mathrm{n})$-reactions by a virtual breakup of the deuteron in the Coulomb field of the nucleus before the actual nuclear interaction takes place. Because of the Coulomb repulsion of the proton this would explain the dominance of (d,p)-reactions. In this context, Oppenheimert also treated the real breakup of the deuteron in the Coulomb field of a nucleus. In the meantime, the subject has developed quite a lot. In addition to the deuteron, many different kinds of projectiles (ranging from light to heavy ions, including radioactive beams) have been used at incident energies ranging from

$\dagger_{\text {to }}$ appear in the proceedings of the RCNP-TMU SYMPOSIUM on Spins in Nuclear and Hadronic Reactions, October 26-28 1999, to be published with World Scientific Publishing Company 
below the Coulomb barrier to medium up to relativistic energies. At higher energies, simplifications arise in the theoretical description, since one can use Glauber theory (or the sudden approximation in a semiclassical framework).

In Chapter 2 we discuss the "spectator breakup" mechanism. The breakup occurs due to the strong interaction of one of the constituents with the target nucleus, while the "spectator" moves on essentially undisturbed. Since this subject has been dealt with extensively in the past $\mathrm{B}$, we wish to give a very brief outline of the development over the last few decades, providing some of the relevant references. (This is of course a biased view of the present authors.) We find that the post-form DWBA is especially suited to treat these processes. We discuss in Chapter 3 Coulomb breakup using this post-form DWBA formalism. This approach has beeen used for a long time for the breakup of the deuteron. Recently, this formalism has also been applied to the breakup of other halo nuclei with a simple structure, like ${ }^{11} \mathrm{Be}$. Also, using the adiabatic (in a sense to be explained below) approach, a formula reminiscent of the formula for the post-form DWBA has recently been developed. The relation between the two formulations will be discussed.

In Chapter 4 we discuss Coulomb dissociation using the semiclassical framework. First and higher order electromagnetic effects are treated. "Postacceleration" can be viewed as a higher order electromagnetic effect. New possibilities for Coulomb dissociation experiments, also in the context of nuclear astrophysics are discussed in Ch. 5. Conclusions and an outlook are given in Chapter 6.

\section{Direct Breakup, Post-Form DWBA}

We assume a three-body model where a target nucleus A interacts with a projectile $a=b+x$. The Hamiltonian is given by

$$
H=T+V_{A x}+V_{A b}+V_{b x} .
$$

This Hamiltonian is decomposed as

$$
H=H_{i}+V_{i} \quad \text { with } \quad H_{i}=T+V_{A a}+V_{b x} \quad \text { and } \quad V_{i}=V_{A x}+V_{A b}-V_{A a}
$$

where an (optical model) interaction $V_{A a}$ is introduced. Another decomposition, which is relevant for the final state, is given by

$$
H=H_{f}+V_{f} \quad \text { with } \quad H_{f}=T+V_{A x}+V_{A b} \quad \text { and } \quad V_{f}=V_{b x} .
$$

We now use the latter decomposition to write the T-matrixelement for the elastic breakup reaction

$$
A+a \rightarrow A+b+x
$$


in the post-form of the DWBA as (see, e.g., eq. 10 of Ref. B)

$$
\begin{aligned}
& T_{\vec{q}_{a} \rightarrow \vec{q}_{b} \vec{q}_{x}}= \\
& \quad \iint d^{3} r_{b x} d^{3} R_{A a} \chi_{\vec{q}_{b}}^{(-) *}\left(\vec{R}_{b-A x}\right) \chi_{\vec{q}_{x}}^{(-) *}\left(\vec{r}_{A x}\right) V_{b x}\left(\vec{r}_{b x}\right) \phi_{a}\left(\vec{r}_{b x}\right) \chi_{\vec{q}_{a}}^{(+)}\left(\vec{R}_{A a}\right),
\end{aligned}
$$

where the $\chi$ 's are the scattering wave functions of $a, b$ and $x$ generated by the appropriate optical potentials. This is quite a complicated expression. Eikonal methods can be used to simplify it. For an intermediate model see, e.g., Ref.t. It contains some simple limits, like the Serber model: see, e.g., Ref. 目. Of course, in the distorted waves of Eq. (5) the interaction of the target with the "participant" as well as the "spectator" is included to all orders in general.

An expression identical to eq. (5) can also be written down in the prior form, but as it stands it would be very complicated to treat numerically, see, e.g., Ref. 目. We want to draw attention to a problem here which is related to the choice of the Hamiltionian in the final state. If the breakup proceeds, e.g., through a resonance $a^{*}$ of the projectile (resonance, or sequential type of breakup) the decomposition eq. (2) would certainly be more appropriate to describe the final state. The interaction $V_{b x}$ should be included in order to properly describe that resonance. If the breakup proceeds directly into the continuum, there seems to be no a priori reason to prefer one or the other decomposition. The two basic mechanisms are shown schematically in Fig. 1. It was found that for Coulomb breakup of the deuteron at low energies, the choice Eq. (3) is distinctly superior. In this way, the Coulomb interaction acts directly on the proton, and not on the centre of mass of the deuteron. For higher beam energies it can be assumed that this distinction will become less and less important. This problem is also reflected in the two choices in Ref. 6 . Yet, this issue persists in general, and further clarification is necessary, also in view of "post-acceleration" to be discussed below.

It is of interest to treat also the case where the subsystem $B=x+A$ can go to other final channels $c$. This is especially simple when the "surface approximation" can be applied: Due to Coulomb repulsion and/or strong absorption the "wave function of the transferred particle"

$$
\int d \xi_{A} \Phi_{B c}^{(-) *} \Phi_{A}=4 \pi \sum_{l_{x} m_{x}} i^{l_{x}} F_{l_{x}}^{c}(r) Y_{l_{x} m_{x}}(\hat{r}) Y_{l_{x} m_{x}}^{*}\left(\hat{q}_{c}\right)
$$

has only to be known in the nuclear exterior. The integration in Eq. (6) is over the $A$ nucleon variables. In this case the overlap integral is given in terms of the S-matrixelement of $x-A$ scattering, which we denote by $S_{l_{x} c}$, as

$$
F_{l_{x}}^{c}(r)=\delta_{l_{x} c} j_{l_{x}}\left(q_{x} r\right)+\sqrt{\frac{m_{x} q_{x}}{m_{c} q_{c}}} \frac{1}{2}\left(S_{l_{x} c}-\delta_{l_{x} c}\right) h_{l_{x}}^{(+)}\left(q_{x} r\right) \quad\left(r \geq R_{0}\right) .
$$




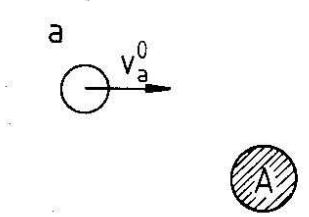

a

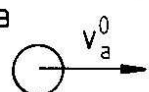

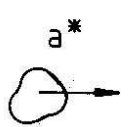

$X$

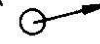

$Q_{b}$

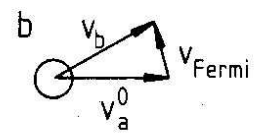

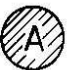

${ }^{x}$

Figure 1: Two basic reaction mechanisms for breakup are shown schematically. In the upper figure, the projectile $a$ is excited to a continuum (resonant) state $a^{*}$ which decays subsequently into the fragments $b$ and $x$. In the lower part substructure $x$ interacts (in all kinds of ways) with the target nucleus $A$, whereas $b=(a-x)$ misses the target nucleus ("spectator"). It keeps approximately the velocity which it had before the collision. [Fig. 1 of Ref. 3 .]

We neglect the spin of the particles, therefore the orbital angular momentum $l_{x}$ is the total angular momentum. For charged particles $x$ the appropriate Coulomb functions have to be used in place of the Hankel functions. The validity of the surface approximation was checked by Kasano and Ichimurat. It was found to be quite good for the (d,p) reaction at $E_{d}=26 \mathrm{MeV}$. Inclusive breakup spectra were measured for many different systems and compared to theory. Agreement is generally good 3 . Of course there are also pre-equilibrium and equilibrium contributions not considered in the inclusive breakup theory. As an example we show in Fig. 2 the $\left({ }^{3} \mathrm{He}, \mathrm{d}\right)$ spectra measured in Osaka.

The theory of inclusive breakup reactions was substantially generalized in a series of papers by M. Ichimura, N. Austern and C. M. Vincent "IAV"). We give two references, from where the full story can be traced back: 10,11 . In this series of papers, also many formal aspects have been deeply elucidated and the relation of post-form to prior-form DWBA (they give identical results) has also been made very clear. Inelastic breakup reactions can also be used to study in an indirect way nuclear reations (much) below the Coulomb barrier ("trojan horse method"), see Ref. 3.12.13.14. In the quasi-free approximation the breakup 


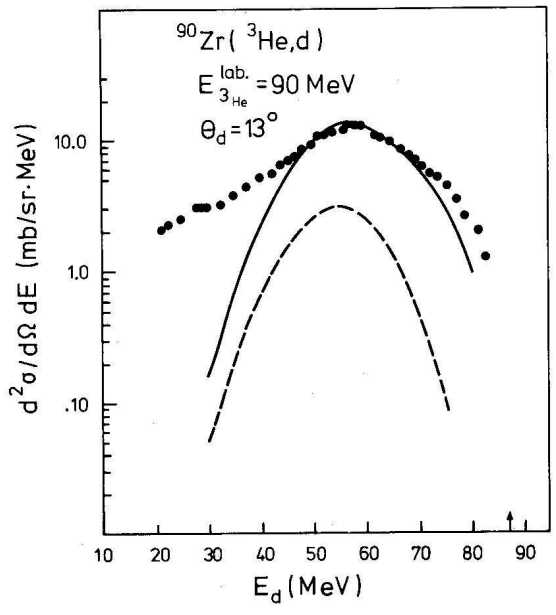

Figure 2: Comparison of experimental ${ }^{90} \mathrm{Zr}\left({ }^{3} \mathrm{He}, \mathrm{d}\right)$ inclusive spectra (Ref. calculations 9 (continuous line) for inclusive breakup. The theoretically calculated contribution due to elastic breakup alone is shown separately by the dashed line. The pnergy corresponding to the three-body threshold is indicated by an arrow. [Fig. 11 of Ref.3.]

cross section can be written as a product of three factors: a) a kinematical factor, $\mathrm{b}$ ) the momentum distribution of particle $x$ in the projectile $a$ and c) an off-shell cross section for the nuclear reaction to be investigated. More general a relation between the S-matrix elements of the two-body cross section and the actually measured three-bady cross section can be established with the help of the surface approximation 4 . In nuclear astrophysics, transfer reactions (like $(\mathrm{d}, \mathrm{p})$ or $\left({ }^{3} \mathrm{He}, \mathrm{d}\right)$, or $\left.(\mathrm{Li}, \alpha)\right)$ are used to study resonant states. E.g., in the ${ }^{22} \mathrm{Na}\left({ }^{3} \mathrm{He}, \mathrm{d}\right)^{23} \mathrm{Mg}$ reaction states near the proton threshold were studied 15. This is relevant for the hydrogen burning of ${ }^{22} \mathrm{Na}$. In principle, also the continuum can be studied. E.g., the "parallelism" of $(\mathrm{d}, \mathrm{p})$ and $(\mathrm{n}, \mathrm{n})$-reactions has been beautifully shown already in 1971, see Ref. 16 . The $d+{ }^{6} \mathrm{Li}$ reaction was investigated in Ref.17 in this indirect way. Another recent application is given in Ref. 18 to the ${ }^{7} \operatorname{Li}(\mathrm{p}, \alpha)^{4} \mathrm{He}$-reaction. An especially interesting case would be the indirect study of the ${ }^{12} \mathrm{C}(\alpha, \gamma){ }^{16} \mathrm{O}$ reaction by means of a $\left({ }^{7} \mathrm{Li}, \mathrm{t}\right)$ or $\left({ }^{6} \mathrm{Li}, \mathrm{d}\right)$ reaction. Quite recently ${ }^{19}$ the sub-Coulomb $\alpha$-transfer reaction $\left({ }^{6} \mathrm{Li}, \mathrm{d}\right)$ and $\left({ }^{7} \mathrm{Li}, \mathrm{t}\right)$ to the bound $2^{+}$and $1^{-}$states in ${ }^{16} \mathrm{O}$ has been used to obtain information on the astrophysical S-factor. 


\section{Spectator Mechanism for Coulomb breakup}

The Coulomb breakup (dissociation) of the deuteron can be viewed as spectator breakup, which was considered in the post-form DWBA in the previous chapter: the Coulomb force acts solely on the proton and the nerttron moves on essentially undisturbed. Using a zero range approximation $\mathrm{E}$, the T-matrixelement separates into a "bremsstrahlung-integral" and a zero range constant. The six-dimensional integral eq. (5) does not separate into two threedimensional integrals. In order to achieve this separation, one can use a "local momentum approximation" to pass from the coordinate $R_{A a}$ (and $R_{b-A x}$ ) to $R_{A x}$ in the distorted wave $\chi_{\vec{q}_{a}}^{(+)}$. (The distorted wave $\chi_{\vec{q}_{b}}^{(-)}$of the neutron becomes a plane wave.) For higher deuteron energies, this should be quite reasonable. In its simplest version, one can use the asymptotic momentum $\vec{q}_{A a}$ as a "local momentum". Now we have achieved a separation of the matrixelement into the bremsstrahlungsintegral $T_{B S}$ and a matrixelement depending on the structure properties of the projectile

$$
T_{\vec{q}_{a} \rightarrow \vec{q}_{b} \vec{q}_{x}} \approx T_{B S}(\vec{Q}) \int d^{3} r_{b x} \exp \left(-i \vec{P} \cdot \vec{r}_{b x}\right) V_{b x}\left(\vec{r}_{b x}\right) \phi_{a}\left(\vec{r}_{b x}\right)
$$

with

$$
\vec{Q}=\frac{m_{A}}{m_{A}+m_{x}} \vec{q}_{b} \quad \text { and } \quad \vec{P}=\vec{q}_{b}-\frac{m_{b}}{m_{b}+m_{x}} \vec{q}_{a} .
$$

Again, a Fourier transform of the ground state wave function of the projeticle multiplied with the interaction potential is obtained.

Applications of this approach to reactions below, around and somewhat above the Coulomb barrier have been extensively made, see, e.g., Ref. 3 . As an example we show in Fig. 3 the p-n coincidence spectra in Subcoulomb deuteron breakup on ${ }^{197} \mathrm{Au}$. A strong "postacceleration effect" can be seen: the average energy of the outgoing proton is much higher than the energy of the neutron. This is due to the Coulomb repulsion. At higher deuteron energies, this effect will be less pronounced. This approach can also be extended to loosely bound neutron halo nuclei, like ${ }^{11} \mathrm{Be} 20$, or ${ }^{19} \mathrm{C}$.

Coulomb dissociation of light nuclei has recently been formulated in an "adiabatic" approach 23.24: It is assumed that projectile excitation is predominantly to states with low excitation energy. One can say that the internal motion is slow as compared to the c.m. motion of the projectile ("frozen nucleus approximation" like in Glauber theory). In this approach, an adiabatic wave function is obtained. This wave function is then inserted into an exact expression for the breakup amplitude. A matrix-element is obtained (eq. 21 of Ref. ${ }^{23}$ ) which is identical to the DWBA matrix-element (8) given abore. Numerical calculations for higher energy deuteron breakup are performed 23 with 


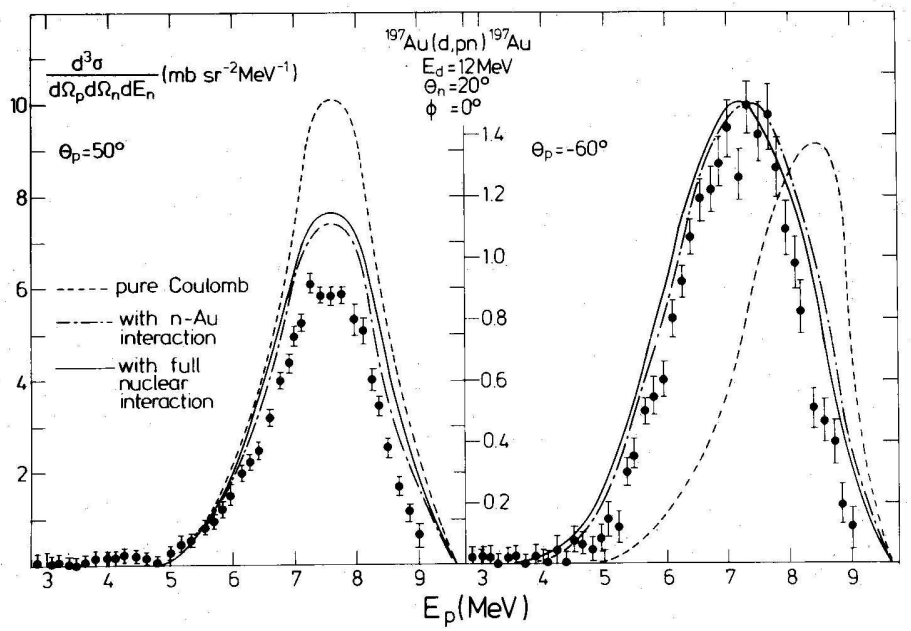

Figure 3: Comparison of calculations 21 for the deuteron breakup epincidence cross section on ${ }^{197} \mathrm{Au}$ at $E_{d}=12 \mathrm{MeV}$ with the experimental results of Ref. 22. A coplanar geometry was used, the neutron angle is $\theta_{n}=20^{\circ}$ and the proton angles $\theta_{p}=50^{\circ}$ and $\theta_{p}=-60^{\circ}$ respectively. It can be seen that, even at sub-Coulomb energies, the neutron-target interaction strongly influences the breakup cross section, whereas the $d+A$ fnd $p+A$ nuclear interactions in the initial and final channel are very small. [Fig. 4 of Ref. 3 .]

good agreement with the data. We note that in this approach as well as in the DWBA method, the Coulomb interaction between $x$ and $A$ is treated nonperturbatively. It is somewhat curious to see that the same formula is obtained by using different approximation schemes. In the following chapter, Coulomb dissociation is treated in the initial state decomposition, eq. (2). These descriptions are more closely connected to the one in this chapter through the post-prior identities as one might first think. It should also be remembered that the approach in this chapter can only work when the neutron is emitted directly into the continuum, with no further interaction with the "core". This works well for the deuteron which has no resonances in the continuum, and probably less well in other cases.

\section{Theory of Electromagnetic Excitation and Dissociation}

\subsection{General}

It is interesting to study nuclear collisions where the colliding nuclei interact only electromagnetically. This can be achieved by using bombarding ener- 
gies below the Coulomb barrier or by choosing very forward scattering angles in high energy collisions. With increasing beam energy states at higher energies can be excited; this can lead, in addition to Coplomb excitation, also to Coulomb dissociation, for a review see, e.g., Ref.25. (Cross-sections for such processes at the forthcoming relativistic heavy ion colliders RHIC at Brookhaven and $\mathrm{LHC}(\mathrm{Pb}-\mathrm{Pb})$ at $\mathrm{CERN} / \mathrm{Geneva}$ are huge 26,27. A new field of studies is opened there, this is, however, beyond the scope of the present review.) Such experiments are also feasible with secondary (radioactive) beams. The electromagnetic interaction which causes the dissociation is well known and therefore there can be a clean interpretation of the experimentat data. This is of interest for nuclear structure and nuclear astrophysics 28,29.30. Multiple electromagnetic excitation can also be important. We mention two aspects: it is a means to excite new nuclear states, like the double phonon giant dipole resonance 30; but it can also be a correction to the one-photon excitation 31.3233. In the equivalent photon approximation the cross section for an electromagnetic process is written as

$$
\sigma=\int \frac{d \omega}{\omega} n(\omega) \sigma_{\gamma}(\omega)
$$

where $\sigma_{\gamma}(\omega)$ denotes the corresponding cross section for the photo-induced process and $n(\omega)$ is called the equivalent photon number. For high enough beam energies it can be well approximated by

$$
n(\omega)=\frac{2}{\pi} Z^{2} \alpha \ln \frac{\gamma v}{\omega R}
$$

where $R$ denotes some cut-off radius. More refined expressions, which take the dependence on multipolarity, beam velgcity or Coulomb-deflection into account, are available in the literature 2,3234 . The theory of electromagnetic excitation is well developed, for nonrelativistic as well as relativistic projectile velocities. In the latter case, an analytical result for all multipolarities was obtained in Ref. 44 . The projectile motion was treated classically in a straightline approximation. Using Glauber theory, the projectile motion can be treated quantally $25,33,35,36,37$. This gives rise to characteristic diffraction effects. The main effect is due to the strong absorption at impact parameters less than the sum of the two nuclear radii.

Nuclear excitation also has to be taken into account. The nuclear breakup of halo nuclei was studied (among others, see e.g. Refs. 38.39 .40 ) in Refs. 36.41. The nuclear interaction of course is less precisely known than the Coulomb interaction. In Ref. 4 the nuclear breakup was studied using the eikonal approximation as well as the Glauber multiple particle scattering theory. No 
Coulomb interaction was included in this approach, as the main focus was on the breakup on light targets. In Ref. 36 on the other hand, the combined effect of both nuclear and Coulomb excitation is studied. The nuclear contribution to the excitation is generally found to be small and has an angular dependence different from the electromagnetic one. This can be used to separate such effects from the electromagnetic excitation.

\subsection{Higher Order Effects}

Higher order effects can be considered in a coupled channels approach, or using higher order perturbation theory. This involves a sum over all intermediate states $n$ considered to be important. Another approach is to integrate the time-denendent Schrödinger equation directly for a given model Hamiltonian 6. $44,45.46 .47$.

If the collision is sudden one can neglect the time ordering in the usual perturbation approach. The interaction can be summed up to infinite order. In order to obtain the excitation cross section, one has to calculate the matrixelement of this operator between the initial and final state (i.e. the intermediate states $n$ do not appear explicitly). A related approach was developed for small values of the adiabaticity parameter $\xi$ (the ratio between collision time and excitation time) in Refs. 31, 32, 33. In a simple zero range model for the neutron-core interaction, analytical results were obtained for $1^{\text {st }}$ and $2^{\text {nd }}$ order electromagnetic excitation 31 . These analytical results can shed light on the higher order effects, which may be present in the Cqulomb dissociation of loosely bound neutron halo nuclei, like ${ }^{11} \mathrm{Be} 48$ and ${ }^{19} \mathrm{C} 49$. Higher order effects were recently discussed by J. A. Tostevin 50. We can use the sudden approximation (corresponding to $\xi=0$ ). Low $\xi$ corrections in $1^{\text {st }}$ and $2^{\text {nd }}$ order can be applied using the formulae given in Ref. 31. Higher order effects decrease strongly (like $1 / b^{4}$ ) withimpact parameter; for grazing collisions the $\xi$-valuegf the RIKEN experiments 43,49 is of the order of 0.1. We can use eq. 33 of Ref. 31 , where higher order effects are fully included. We note the following points: for the angle integrated cross-section the $2^{\text {nd }}$ order leads to an increase, whereas the interference of $1^{\text {st }}$ and $3^{r d}$ order leads to a decrease, an effect also found by J. A. Tostevin. Of course, in the expression for the sudden approximation all these higher order effects are included automatically. It would be nice to have some numerical results, say for the ${ }^{11} \mathrm{Be}$ or ${ }^{19} \mathrm{C}$ systems 48,49 . It can be supposed that they would match closely to the results of Ref.50. This is because the same physics is described, and higher order electromagnetic effects are included to all orders in both approaches. More work can certainly clarify this issue. 


\subsection{Some Results}

In this context it is also worthwhile to remember that electromagnetic excitation of intermediate energy (exotic) beams has been recently developed into a useful spectroscopic tool 51,52. By measuring the excitation energies of the first $2^{+}$states and the corresponding B(E2)-values, nuclear structure effects like deformation, can be studied in a unique way for nuclei far off stability. Electromagnetic excitation of the $1^{\text {st }}$ excited state in ${ }^{11} \mathrm{Be}$ has been studied experimentally at GANIL53, RIKEN 54 and MSUE5. This is a good test case, since the $\mathrm{B}(\mathrm{E} 1)$-value of the corresponding ground-state transition is known already. Theoretical calculations 5.57 show that higher order effects are expected to be small.

Coulomb dissociation of exotic nuclei is a valuable tool to determine electromagnetic matrix-elements between the ground state and the nuclear continuum. The excitation energy spectrum of the ${ }^{10} \mathrm{Be}+\mathrm{n}$ system in the Coulomb dissociation of the one-neutron halo nucleus ${ }^{11} \mathrm{Be}$ on a $\mathrm{Pb}$ target at $72 \mathrm{~A} \mathrm{MeV}$ was measured 18 . Low lying E1-strength was found. The Coulomb dissociation of the extremely neutron-rich nucleus ${ }^{19} \mathrm{C}$ was recently studied in a similar way 4 . The neutron separation energy of ${ }^{19} \mathrm{C}$ could also be determined to be $530 \pm 130 \mathrm{keV}$. Quite similarly, the Coulomb dissociation of the $2 \mathrm{n}$-halo nuclems ${ }^{11} \mathrm{Li}$ was studied in various laboratories 58,59.60. In an experiment at MSU61, the correlations of the outgoing neutrons were studied. Within the limits of experimental accuracy, no correlations were found.

In nuclear astrophysics, radiative capture reactions of the type $b+c \rightarrow a+\gamma$ play a very important role. They can also be studied in the time-reversed reaction $\gamma+a \rightarrow b+c$, at least in those cases where the nucleus $a$ is in the ground state. As a photon beam, we use the equivalent photon spectrum which is provided in the fast peripheral collision. Reviews both from an experimental as well as theoretical point of view have been given 28 , so we want to concentrate here on a few points.

The ${ }^{6} \mathrm{Li}$ Coulomb dissociation into $\alpha+\mathrm{d}$ has been a test case of the method, see Ref.28. The ${ }^{7} \mathrm{Be}(\mathrm{p}, \gamma)^{8} \mathrm{~B}$ radiative capture reaction is relevant for the solar neutrino problem. It determines the production of ${ }^{8} \mathrm{~B}$ which leads to the emission of high energy neutrinos. There are direct reaction measurements, for a recent one see Refs. 6263 Coulomb dissociation of ${ }^{8} \mathrm{~B}$ has been studied at RIKEN64, MSU65 and GSI 66 64. Theoretical calculations are shown in Fig. 4. It is seen that E1 excitation is large and peaked at very forward angles. E2 excitation is also there, with a characteristically different angular distribution. Nuclear diffraction effects are small. Altogether it is quite remarkable that completely different experimental methods with different possible systematic 

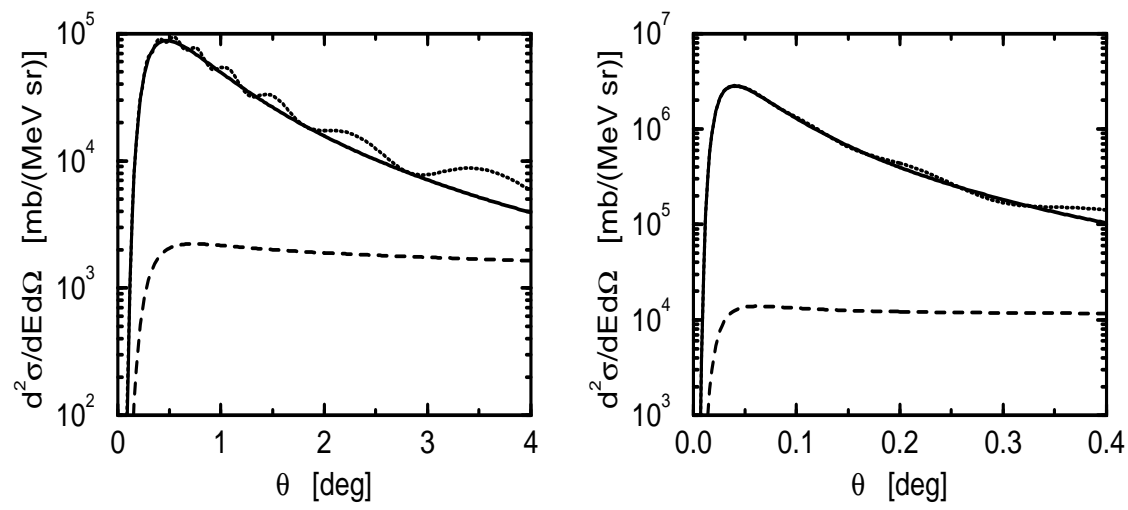

Figure 4: Coulomb dissociation cross section of ${ }^{8} \mathrm{~B}$ scattered on ${ }^{208} \mathrm{~Pb}$ as a function of the scattering angle for projectile energies of $46.5 A \cdot \mathrm{MeV}$ (left) and $250 A \cdot \mathrm{MeV}$ (right) and a ${ }^{7} \mathrm{Be}-\mathrm{p}$ relative energy of $0.3 \mathrm{MeV}$. First order results E1 (solid line), E2 (dashed line) md E1+E2 excitation including nuclear diffraction (dotted line). [From figs. 4 and 5 of Ref. 33 .]

errors lead to results that are quite consistent.

\section{Possible new applications of Coulomb dissociation for nuclear astrophysics}

Nucleosynthesis beyond the iron peak proceeds mainly by the $\mathrm{r}$ - and s-processes (rapid and slow neutron capture) 68.60. To establish the quantitative details of these processes, accurate energy-averaged neutron-capture cross sections are needed. Such data provide information on the mechanism of the neutroncapture process and time scales, as well as temperatures involved in the process. The data should also shed light on neutron sources, required neutron fluxes and possible sites of the processes (see Ref. 68). The dependence of direct neutron capture on nuclear structure models was investigated in Ref.70. The investigated models yield capture cross-sections sometimes differing by orders of magnitude. This may also lead to differences in the predicted astrophysical r-process paths. Because of low level densities, the compound nucleus model will not be applicable.

With the new radioactive beam facilities (either fragment separator or ISOL-type facilities) some of the nuclei far off the valley of stability, which are relevant for the r-process, can be produced. In order to assess the r-process path, it is important to know the nuclear properties like $\beta$-decay half-lifes and neutron binding energies. Sometimes, the waiting point approximation 68.69 is 
introduced, which assumes an $(\mathrm{n}, \gamma)$ - and $(\gamma, \mathrm{n})$-equilibrium in an isotopic chain. It is generally believed that the waiting point approximation should be replaced by dynamic r-process flow calculations, taking into account $(\mathrm{n}, \gamma),(\gamma, \mathrm{n})$ and $\beta$-decay rates as well as time-varying temperature and neutron density. In slow freeze-out scenarios, the knowledge of $(\mathrm{n}, \gamma)$ cross sections is important.

In such a situation, the Coulomb dissociation can be a very useful tool to obtain information on $(\mathrm{n}, \gamma)$-reaction cross sections on unstable nuclei, where direct measurements cannot be done. Of course, one cannot and need not study the capture cross section on all the nuclei involved; there will be some key reactions of nuclei close to magic numbers. It was proposed 11 to use the Coulomb dissociation method to obtain information about $(\mathrm{n}, \gamma)$ reaction cross sections, using nuclei like ${ }^{124} \mathrm{Mo},{ }^{126} \mathrm{Ru},{ }^{128} \mathrm{Pd}$ and ${ }^{130} \mathrm{Cd}$ as projectiles. The optimum choice of beam energy will depend on the actual neutron binding energy. Since the flux of equivalent photons has essentially an $\frac{1}{\omega}$ dependence, low neutron thresholds are favourable for the Coulomb dissociation method. Note that only information about the $(\mathrm{n}, \gamma)$ capture reaction to the ground state is possible with the Coulomb dissociation method. The situation is reminiscent of the loosely bound neutron-rich light nuclei, like ${ }^{11} \mathrm{Be},{ }^{11} \mathrm{Li}$ and ${ }^{19} \mathrm{C}$.

In Ref. 31 the $1^{\text {st }}$ and $2^{\text {nd }}$ order Coulomb excitation amplitudes are given analytically in a zero range model for the neutron-core interaction (see section 4.2). This can be very useful to assess how far one can go down in beam energy and still obtain meaningful results with the Coulomb dissociation method. I.e., where the $1^{\text {st }}$ order amplitude can still be extracted experimentally without being too much disturbed by corrections due to higher orders. For future radioactive beam facilities, like ISOL od SPIRAL, the maximum beam energy is an important issue. We propose to use the handy formalism of Ref. 31 to assess, how far one can go down in beam energy. For Coulomb dissociation with two charged particles in the final state, like in the ${ }^{8} \mathrm{~B} \rightarrow{ }^{7} \mathrm{Be}$ $+\mathrm{p}$ experiment with a $26 \mathrm{MeV}^{8} \mathrm{~B}$ beam 72 such simple formulae seem to be unavailable and one should resort to the more involved approaches mentioned in section 4.2 .

A new field of application of the Coulomb dissociation method can be two nucleon capture reactions. Evidently, they cannot be studied in a direct way in the laboratory. Sometimes this is not necessary, where the relevant information about resonances involved can be obtained by other means (transfer reactions, etc.), like in the triple $\alpha$-process.

Tyg-neutron capture reactions in supernovae neutrino bubbles are studied in Ref.73. In the case of a high neutron abundance, a sequence of two-neutron capture reactions, ${ }^{4} \mathrm{He}(2 \mathrm{n}, \gamma){ }^{6} \mathrm{He}(2 \mathrm{n}, \gamma){ }^{8} \mathrm{He}$ can bridge the $A=5$ and 8 gaps. The ${ }^{6} \mathrm{He}$ and ${ }^{8} \mathrm{He}$ nuclei may be formed preferentially by two-step resonant 
processes through their broad $2^{+}$first expited states 73. Dedicated Coulomb dissociation experiments can be useful,see 4 . Another key reaction can be the ${ }^{4} \mathrm{He}(\alpha \mathrm{n} \gamma)$ reaction $\mathrm{3}$. The ${ }^{9} \mathrm{Be}(\gamma, \mathrm{n})$ reaction has been studied directly (see Ref. .5 .76 ) and the low energy $s \frac{1}{2}$ resonance is clearly established. Despite this, a ${ }^{9} \mathrm{Be}$ Coulomb dissociation experiment could be rewarding (cf. also Ref. $\frac{77}{7}$ ). Other useful information is obtained from (e,e') and (p,p') reactions on ${ }^{9} \mathrm{Be} 7 \mathrm{7}$.

In the rp-process, two-proton capture reactions can bridge the waiting points 69 .80. From the ${ }^{15} \mathrm{O}(2 \mathrm{p}, \gamma){ }^{17} \mathrm{Ne},{ }^{18} \mathrm{Ne}(2 \mathrm{p}, \gamma){ }^{20} \mathrm{Mg}$ and ${ }^{38} \mathrm{Ca}(2 \mathrm{p}, \gamma){ }^{40} \mathrm{Ti}$ reactions considered in Ref. 80 , the latter can act as an efficient reaction link at conditions typical for X-ray bursts on neutron stars. A ${ }^{40} \mathrm{Ti} \rightarrow \mathrm{p}+\mathrm{p}+$ ${ }^{38} \mathrm{Ca}$ Coulomb dissociation experiment should be feasible. The decay with two protons is expected to be sequential rather than correlated ("2 $\mathrm{H}$ "-emission). The relevant resonances are listed in Table XII of Ref. 80 . In Ref. 81 it is found that in X-ray bursts $2 \mathrm{p}$-capture reactions accelerate the reaction flow into the $Z \geq 36$ region considerably. In Table 1 of Ref. 81 nuclei on which $2 \mathrm{p}$-capture reactions may occur, are listed; the final nuclei $2 \mathrm{re}{ }^{68} \mathrm{Se},{ }^{72} \mathrm{Kr},{ }^{76} \mathrm{Sr},{ }^{80} \mathrm{Zr},{ }^{84} \mathrm{Mo}$,

${ }^{88} \mathrm{Ru},{ }^{92} \mathrm{Pd}$ and ${ }^{96} \mathrm{Cd}$ (see also Fig. 8 of Ref. ${ }^{99}$ ). It is proposed to study the Coulomb dissociation of these nuclei in order to obtain more direct insight into the $2 \mathrm{p}$-capture process.

\section{Conclusion}

We discussed essentially two types of breakup mechanisms. However, we saw that they are somehow related, although more work should be still done to clarify this in more detail. The spectator mechanism is also useful as an indirect method to study astrophysically relevant reactions below the Coulomb barrier. This is much in the same way as transfer reactions have been traditionally used to study spectroscopic factors. Peripheral collision of medium and high energy nuclei (stable or radioactive) passing each other at distances beyond nuclear contact and thus dominated by electromagnetic interactions are important tools of nuclear physics research. The intense source of quasireal (or equivalent) photons has opened a wide horizon of related problems and new experimental possibilities especially for the present and forthcoming radioactive beam facilities to investigate efficiently photo-interactions with nuclei (single- and multiphoton excitations and electromagnetic dissociation).

\section{Acknowledgments}

We have enjoyed collaboration and discussions on the present topics with very many people; too many to name them all. We especially wish to mention C. A. Bertulani, H. Rebel, F. Rösel, and R. Shyam. One of us (G.B.) wishes 
to thank his Japanese hosts for the kind invitation to the RCNP/TMU symposium, he is also very grateful for their generous financial support.

\section{References}

1. J. R. Oppenheimer, Phys. Rev. 47, 845 (1935)

2. J. R. Oppenheimer and M. Phillips, Phys. Rev. 48, 500 (1935)

3. G. Baur, F. Roesel, D. Trautmann and R. Shyam, Phys. Rep. 111, 333 (1984)

4. A. Bonaccorso, Phys. Rev C 60, 054602 (1999)

5. G. Baur and D. Trautmann, Phys. Reports 25C, 293 (1976)

6. V. S. Melezhik and D. Baye, Phys. Rev. C 59, 3232 (1999)

7. A. Kasano and M. Ichimura, Phys. Lett. B 115, 81 (1982)

8. N. Matsuoka, A. Shimizu, K. Hosono, T. Saito, M. Kondo, H. Sakaguchi, A. Goto, and F. Ohtani, Nucl. Phys. A 337, 269 (1980)

9. R. Shyam, G. Baur, F. Rösel, and D. Trautmann, Phys. Rev. C 22, 1401 (1980)

10. M. Ichimura, N. Austern and C. M. Vincent, Phys. Rev. C 37, 2264 (1988)

11. M. Ichimura, Theory of Inclusive Break-up Reactions, Invited talk at: International Conference on Nuclear Reaction Mechanism, January 3-9, 1989, Calcutta, India

12. G. Baur, Phys. Lett. B 178,135 (1986)

13. G. Baur, Proceedings of the Varna Conference 1985, Nuclear Energy 25, Sofia, Bulgarian Academy of Sciences (1987)

14. S. Typel and H. H. Wolter, submitted to Few-Body Systems

15. S. Schmidt, C. Rolfs, W. H. Schulte, H. P. Trautvetter, R. W. Kavanagh, C. Hategan, S. Faber, B. D. Valnion and G. Graw, Nucl. Phys. A 591, 227 (1995)

16. H. Fuchs, H. Homeyer, Th. Lorenz, H. Oeschler, Phys. Lett. B 37, 285 (1971)

17. S. Cherubini, V. N. Kondratyev, M. Lattuada, C. Spitaleri, D. Miljanic, M. Zadro and G. Baur, Astrophys. J. 457855 (1996)

18. C. Spitaleri et al., Phys. Rev. C 60, 055802 (1999)

19. C. R. Brune et al., Phys. Rev. Lett. 83, 4025 (1999)

20. R. Shyam, P. Banerjee and G. Baur, Nucl. Phys. A 540, 341 (1992)

21. G. Baur and D. Trautmann, Phys. Lett. B 42, 31 (1972).

22. L. Jarczyk, J. Lang, R. Müller, D. Balzer, P. Viatte, and P. Marmier, Phys. Rev. C 8, 68 (1973)

23. J. A. Tostevin, S. Rugmai and R. C. Johnson, Phys. Rev. C 57, 3225 
(1998)

24. P. Banerjee, I. J. Thompson and J. A. Tostevin, Phys. Rev. C 58, 1042 (1998)

25. C. A. Bertulani and G. Baur, Phys. Rep. 163, 299 (1988)

26. G. Baur and C. A. Bertulani, Nucl. Phys. A 505, 835 (1989)

27. K. Hencken, D. Trautmann and G. Baur, Z. Phys. C 68, 473 (1995); G. Baur, K. Hencken and D. Trautmann, J. Phys. G: Nucl. Part. Phys. 24, 1657 (1998)

28. G. Baur and H. Rebel, J. Phys. G: Nucl. Part. Phys. 20, 1 (1994) and Ann. Rev. Nucl. Part. Sci. 46, 321 (1996)

29. J. Vervier, Prog. Part. Nucl. Phys. 37, 435 (1996)

30. G. Baur and C. A. Bertulani, Phys. Lett. B 174, 23 (1986)

31. S. Typel and G. Baur, Nucl. Phys. A 573, 486 (1994)

32. S. Typel and G. Baur, Phys. Rev. C 50, 2104 (1994)

33. S. Typel, H. H. Wolter and G. Baur, Nucl. Phys. A 613, 147 (1997)

34. A. Winther and K. Alder, Nucl. Phys. A 319, 518 (1979)

35. C. A. Bertulani and A. M. Nathan, Nucl. Phys. A 554, 158 (1993)

36. A. Muendel and G. Baur, Nucl. Phys. A 609, 254 (1996)

37. G. Baur and C. A. Bertulani, Phys. Rev. C 56, 581 (1997)

38. C. A. Bertulani and K. W. McVoy, Phys. Rev. C 46, 2638 (1992)

39. P. Banerjee and R. Shyam, Phys. Lett. B 349, 421 (1995)

40. F. Barranco, E. Vigezzi, and R. A. Broglia, Z. Phys. A 356, 45 (1996)

41. K. Hencken, G. Bertsch and H. Esbensen, Phys. Rev. C 54, 3043 (1996)

42. R. Serber, Phys. Rev. 72, 1008 (1947)

43. R. J. Glauber, Phys. Rev. 99, 1515(1955)

44. H. Esbensen, G. F. Bertsch and C. A. Bertulani, Nucl. Phys. A 581, 107 (1995)

45. H. Utsunomia, Y. Tokimoto, T. Yamagata, M. Ohta, Y. Aoki, K. Hirota, K. Ieki, Y. Iwata, K. Katori, S. Hamada, Y.-W. Lui, R. P. Schmitt, S. Typel and G. Baur, Nucl. Phys. A 654, 928c (1999)

46. S. Typel, H. H. Wolter, Z. Naturforsch. 54 a, 63 (1999)

47. T. Kido, K. Yabana and Y. Suzuki, Phys. Rev. C 50, R1276 (1994)

48. T. Nakamura et al., Phys. Lett. B 331, 296 (1994)

49. T. Nakamura et al., Phys. Rev. Lett. 83, 1112 (1999)

50. J. A. Tostevin, Paper presented at : 2nd International Conference on Fission and Neutron Rich Nuclei, St. Andrews, Scotland, June 28 - July 2 1999, to be published by World Scientific

51. T. Motobayashi et al., Phys. Lett. B 346, 9 (1995)

52. H. Scheit et al., Phys. Rev. Lett. 77, 3967 (1996)

53. R. Anne et al., Z. Phys. A 352, 397 (1995) 
54. T. Nakamura et al., Phys. Lett. B 394, 11 (1997)

55. M. Fauerbach et al., Phys. Rev. C 56, R1 (1997)

56. C. A. Bertulani, L. F. Canto and M. S. Hussein, Phys. Lett. B 353, 413 (1995)

57. S. Typel and G. Baur, Phys. Lett. B 356, 186 (1995)

58. T. Kobayashi et al., Phys. Lett. B 232, 51 (1989)

59. S. Shimoura et al., Phys. Lett. B 348, 29 (1995)

60. M. Zinser et al., Nucl. Phys. A 619, 151 (1997)

61. K. Ieki, A. Galonski et al., Phys. Rev. C 54, 1589 (1996)

62. G. Bogoert et al., presented at SOLAR Neutrino Conference IV, Heidelberg, April 1997

63. F. Hammache et al., Phys. Rev. Lett. 80, 928 (1998)

64. T. Motobayashi et al., Phys. Rev. Lett. 73, 2680 (1994)

65. J. H. Kelley et al., Phys. Rev. Lett. 77, 5020 (1996)

66. K. Sümmerer et al., GSI Darmstadt, experimental proposal and private communication

67. N. Iwasa et al., Phys. Rev. Lett. 83, 2910 (1999)

68. C. E. Rolfs and W. S. Rodney, Cauldrons in the Cosmos, The University of Chicago Press (1988)

69. J. J. Cowan, F.-K. Thielemann and J. W. Truran, Phys. Rep. 208, 267 (1991)

70. T. Rauscher et al., Phys. Rev. C 57, 2031 (1998)

71. M. Gai, ISOL workshop, Columbus/Ohio, July 30 - August 1, 1997

72. J. von Schwarzenberg et al., Phys. Rev. C 53, R2598 (1996)

73. J. Görres, H. Herndl, I. J. Thompson and M. Wiescher, Phys. Rev. C 52, $2231(1995)$

74. T.Aumann et al. Phys.Rev.C 59 (1999) 1252

75. F. Ajzenberg-Selove, Nucl. Phys. A 490, 1 (1988)

76. H.Utsunomya et al. "Photoneutron cross section measurement on ${ }^{9} \mathrm{Be}$ using laser induced Compton backscattered photons" to be published in the proceedings of the International Symposium on Physics with Storage Rings (STORI99) Bloomington, Indiana, September 12-16,1999

77. D. M. Kalassa and G. Baur, J. Phys. G: Nucl. Part. Phys. 22, 115 (1996)

78. G. Kuechler, A. Richter and W. von Witsch, Z. Phys. A 326, 447 (1987)

79. NuPECC Report, Nuclear and Particle Astrophysics, July 16, 1997, I. Baraffe et al., F.-K. Thielemann (convener)

80. J. Görres, M. Wiescher and F.-K. Thielemann, Phys. Rev. C 51 (1995) 392

81. H. Schatz et al., Phys. Rep. 294, 167 (1998) 